\title{
Postoperative TKA alignment in sagittal and axial plane, a systematic review
}

\author{
Bogdan Ştefan Crețu* **, Călin Dragosloveanu *, Dragoş Cotor *, \\ Şerban Dragosloveanu ***, Cristian loan Stoica* ** \\ * "Foişor" Orthopaedics-Traumatology and Osteoarticular TB Hospital, Bucharest, Romania; \\ * * "Carol Davila" University of Medicine and Pharmacy, Bucharest, Romania
}

Correspondence to: Bogdan Ştefan Crețu, MD, PhD, "Foişor" Orthopaedics-Traumatology and Osteoarticular TB Hospital, Bucharest, 35-37 Ferdinand I Blvd., Bucharest, Romania, Mobile phone: +40741 127 187, E-mail: jfrbogdan@yahoo.com

\begin{abstract}
The aim of this paper was to review the existing described methods for measuring postoperative TKA alignment in sagittal and axial plane and to review the existing literature regarding the axial plane evaluation with the use of the computer tomography. The most frequent mistakes when positioning the total knee arthroplasty (TKA) components are done in the axial plane, so it is necessary to know what the limits of the radiographic evaluation are and, for this evaluation, the CT scan is the most valuable in assessing the rotation of the components.

Keywords: TKA alignment, sagittal plane, axial plane, rotational analysis, CT evaluation
\end{abstract}

\section{Introduction}

The sagittal and axial preoperative and postoperative evaluations of the total knee arthroplasty are essential steps in improving the surgical outcome. Precise positioning of the patient is crucial for obtaining correct radiological images. In the sagittal plane, the anatomical and mechanical axis of the femur and tibia, the femoral offset evaluation, patellar height, the joint line height, and the patellar tendon angle are important factors for the preoperative planning and for evaluating the positioning of the TKA components. In the axial plane, only the femur can be evaluated. The axial plane evaluation for the total knee arthroplasty may be done by using CT scan or
MRI. The CT is the most valuable examination in assessing the rotation of the components.

\section{Evaluation of the knee in sagittal plane (lateral)}

\section{Patient positioning}

The precise positioning of the patient is crucial for obtaining the radiological image in sagittal plane. This image can be obtained with or without weight bearing of the lower limb. The knee is flexed at $30^{\circ}$, with the patella perpendicular on the cassette and with the lower limb parallel to the radiological table. The contralateral limb is positioned in a slightly external rotation, allowing enough lateral space for the affected knee [1]. For the lateral 
weight bearing radiography, the knee is flexed at $3^{\circ}$; the contralateral lower limb is positioned posterior, the patient being in a semi flexion position of the knee.

Both images are likely obtained after a fluoroscopically exploration that certifies the good position of the knee.

After a good positioning of the knee on the sagittal plane, the condyles will appear overlapped. The curve of the femur and tibia must be included in the image as much as possible, obtained with a $24 \times 30 \mathrm{~cm}$ image (10x12 inch). The patella must be tangent to the beam to determine the height relative to the joint $[1,2]$. The fluoroscopically examination helps obtaining the femoral condyles overlap for a correct measurement of the position of the components. If the fluoroscopy is not available, an external rotation of $3^{0}-5^{0}$ can be done to try to obtain femoral condyles overlap [3]. This image is obtained using a $24 \times 30 \mathrm{~cm}$ film (10x12 inch), the beam is on $5^{\circ}$ cranial direction, placed at $97 \mathrm{~cm}$ (39 inch) from the cassette and centered between patellar apex and $1 \mathrm{~cm}$ distal from the medial condyle.

Paley proposed the use of a 71 or $91 \mathrm{~cm}$ cassette with the beam centered knee joint at $305 \mathrm{~cm}$ (10 feet) distance from the patient for the lateral weight-bearing image of the lower $\operatorname{limb}[3]$.

Chung et al. created a new technique for obtaining a lateral image of the whole femur in which the femoral head is viewed clearly and both femoral condyles are overlapped [7]. The thigh of the patient is positioned on a $17 \times 17$ inch digital detector and the $X$-ray beam is angled at $15^{0}$ cranial.

\section{Axis evaluation}

The femoral anatomical and mechanical axis

The sagittal plane radiology is based on data obtained on lateral short film (14X17 inch). Sagittal anatomical angle of the femoral component is named "femoral flexion" (gamma angle). The value of this angle is given by the sagittal anatomical axis of the femur and sagittal axis of femoral component.

The sagittal anatomical axis of the femur on the lateral view is obtained uniting the furthest point from the middle of the femoral shaft with a second point placed at $10 \mathrm{~cm}$ proximal to the joint line in the middle of the femoral shaft. The sagittal axis of the femoral component is perpendicular on the distal condyle plane of the implant that can be represented by the distal resection of the femur or by the intercondylar notch of the implant.

The sagittal mechanical axis of the femur is an axis drawn from the center of the femoral head, identified using the Mose circles, and the center of the femoral component on the sagittal plane $[4,5]$.

There are 2 possibilities to identify the sagittal mechanical axis of the femur by the distal points:

- Mechanical axis 1 is a line that goes from the center of the femoral head to a point that is placed $1 \mathrm{~cm}$ anterior to the Blumensaat line (a line which goes through the intercondylar notch on the lateral view)

- Mechanical axis 2 is defined as a line that goes from the center of the femoral head to a point identified $65 \%$ posterior on a line between the anterior cortex and the most prominent point from the posterior medial condyle.

The sagittal anatomical axis of the femur is obtained by drawing a line through the proximal, middle and distal centers of the femoral shaft on a whole lateral lower limb radiography $[3,6]$. This produces a segmented line, which considers the sagittal femoral curve (between $4^{0}$ and $9^{\circ}$ ) $[7,8]$.

The distal sagittal anatomical axis of the femur:

- "Distal anterior cortex axis" is defined as a line, which connects two points on the anterior cortex at $5 \mathrm{~cm}$ and $10 \mathrm{~cm}$ proximal to the joint line.

- "Distal medullary axis"

A study found an angular difference average of $2^{0}\left(0^{0}-4^{0}\right)$ between the mechanical axis and 
the sagittal anatomical axis of the distal femur. Far as $1^{0}$ of anterior femoral curve, the angular deviation between those two axes grows for about $0,5^{\circ}[7]$. The anterior cortical axis has an average value to the mechanical axis of $4^{\circ}\left(0^{\circ}\right.$ 110) [8].

The evaluation of the position in the sagittal plane of the femoral component must consider the anatomy of the distal third of the femur and the neutral position of the femoral component that has the distal condyles perpendicular on the anatomical axis of the distal femur [2].

The sagittal anatomical and mechanical axis of the tibia

Regarding the tibia, the angle called "tibial angle" as well is the equivalent of the so-called "tibial sloping". This angle is an anatomical angle on that sagittal plane, which is formed between a tangent line at the base of the tibial component and a sagittal tibial anatomical line that is formed by connecting the furthest point of the center of the tibial shaft with a point located $10 \mathrm{~cm}$ under the knee joint, in the middle of the tibial shaft [3,9-13].

There are 5 anatomical landmarks described for the sagittal anatomical line of the tibia [14]:

- The anterior cortical line of the tibia (acl) is a line, which connects two points on the tibial cortex that are placed proximal and anterior at 5 and $15 \mathrm{~cm}$ distally from the joint line.

- The proximal anatomical axis (paa).

- The central anatomical axis (caa).

- The posterior cortical line (pcl) of the proximal tibia is a line that connects two points of the posterior cortex of the tibia, which are placed on the posterior cortex at $5 \mathrm{~cm}$ and 15 $\mathrm{cm}$ distally from the joint line.

- Fibular shaft axis is a line that connects the distal and proximal parts of the fibular shaft.

The normal tibial sloping is between $5^{\circ}$ and $11^{0}[15,16]$.

\section{The femoral offset evaluation}

The posterior condylar offset (PCO)

Is the maximal thickness of the posterior condyles, projected posteriorly by the posterior cortical tangent of the femoral shaft for the anatomical knee and for the knee prosthesis [17]. There is a great correlation between regaining the $\mathrm{PCO}$ and the maximum flexion, the more the PCO decreases after surgery, the more the flexion decreases. For every $2 \mathrm{~mm}$ of PCO decreasing, the flexion decreases for about $12.2^{\circ}$. Regaining the $P C O$ is important in total knee arthroplasty with preserving the posterior cruciate ligament. Soda et al. proposes a new parameter called PCO rate [18]. Arabori et al. found a correlation between PCO and flexion only in cases with total knee arthroplasty with preserved posterior cruciate ligament [19].

\section{The anterior condylar offset (ACO)}

Is the maximal thickness of the anterior condyles, projected anterior by the anterior cortical tangent of the femoral shaft for the normal knee and for the knee prosthesis as well $[20,21]$. After intraoperative measurements in total knee arthroplasty, the standard ACO value represented by the anterior resection of 10.9 $\mathrm{mm}$ for a males and $10.1 \mathrm{~mm}$ for females [22]. If the ACO increases postoperative, a pressure increasing in patellofemoral articulation may appear with a limitation of postoperative movement [23]. Increasing the ACO goes to an increase of the trochlear groove height in the anterior compartment, the extension arc of the knee increases and in the end, the flexion decreases. After a total knee arthroplasty, Miller et al. discovered an increase of lateral and medial flanges by $1.1 \pm 2.6 \mathrm{~mm}$ and $0.5 \pm 2.2$ $\mathrm{mm}$, respectively and the trochlear groove by $0 \pm 1.1 \mathrm{~mm}$ [24]. They calculated that an increase between 2 and $4 \mathrm{~mm}$ of the anterior cortex, the flexion decreases by $1.8^{\circ}$ and $4.4^{\circ}$, respectively. The modification of the shape of the anterior femur has few consequences on the passive flexion but the clinical implications on the patient's symptoms remain unidentified [24].

\section{Patellar height}

Between all the indexes used for the patellar 
measurement after total knee arthroplasty, just those that are related to the tibia (IS, CD, BP) can be used [25-27]. Referring to the femur is not possible after arthroplasty (Blumennsatt, Bernageau) $[28,29]$.

Insall-Salvati Index is the ratio between the length of the patellar tendon, measured on the posterior surface from de distal pole of the patella to the insertion on the tibial tubercle and the length of the patella measured on the greatest diagonal line drawn across the patella. A normal value is 1.02 with a variation of less than $20 \%$. A ratio higher than 1.2 indicates a patella alta and a ratio lower than 0.8 indicates a patella baja [27].

Blackbourne-Peel Index is the ratio between A line, represented by the perpendicular distance from the inferior articular edge of the patella and the joint line and a B line represented by the length of the articular surface of the patella. The normal value is 0.8 (0.65-1.38). A ratio bigger than 1.2 indicates patella alta, a; lower ratio indicates patella baja [25].

Caton-Deschamps Index is the ratio between the distance from the inferior margin from the articular surface of the patella to the superior margin of the tibial plateau (A) and the length of the patellar articular surface (B). The normal value is 1.0 (0.8-1.2). A ratio higher than 1.3 indicates patella alta, and an index lower than 0.7 indicates patella baja [26].

The IS has some disadvantages: The tibial insertion of the tendon is hard to identify and the length of the patella does not show the length of the articular surface.

The modified Caton index was proposed by Aglietti et al., being considered the most suitable for the measurement of the patellar height in the prosthetic knee [30].

\section{The joint line height}

Is defined as a line that passes distally by the femoral condyles. The level of the condylar surfaces depends on the spontaneous flexion of the articulation. The position of the joint line is the distance (average of $2.2 \mathrm{~cm}$ ) from the proximal edge of the tibial tuberosity to the joint line $[10,18]$. After total knee arthroplasty, the modification of the joint line position is measured as a difference between the preoperative and postoperative status of the joint line. This value is negative if the joint line is lower and positive if the joint line is higher [31]. For the cases in which the tibial tubercle is not well defined, the usage of the tip of the peroneal head would be a proper method [8].

Usually, the joint line is found $15.4 \pm 5.4 \mathrm{~mm}$ above the head of the peroneus, in the sagittal plane and $13.9 \pm 5.8 \mathrm{~mm}$ in coronal plane $[32,33]$.

The position of the joint line is a complex tridimensional concept and it needs a CAT scan for a correct evaluation.

\section{The patellar tendon angle}

The value of this angle allows the analysis of the knee kinetics in weight bearing position with or without sagittal plane [24]. It is an angle represented by the patellar tendon and the tibial axis. There is a correlation between this angle and the flexion angle of the knee, which may be quantified [24]. With this angle, a good analysis of the patellofemoral and tibiofemoral kinetics can be made. Major modifications of these angles are due to some abnormalities between tibia and femur. Anterior subluxation of the femur increases the angle value and the posterior subluxation decreases the angle. The patellar tendon angle in a normal knee has an average of $20^{\circ}$ in extension position. In flexion, the angle decreases in a linear fashion: $0^{0}$ at $80^{\circ}$ of flexion and $10^{\circ}$ at $120^{\circ}$ of flexion $[24,34,35$ ].

\section{Axial plane knee evaluation}

\section{Patient positioning for patellar views}

There are many positions described by different authors (Laurin, Ficat, Merchant) [3638].

1. Ficat and Hungerford: The patient's knees 
are flexed at the end of the radiological table. The tube is positioned at the legs of the patient and the cassette on the anterior thigh. In this position, the knee is perpendicular on the beam. It can be made at $30^{\circ}, 60^{\circ}$, and $90^{\circ}$.

2. Laurin: The patient is positioned on the radiological table with the legs close to the edge. The beam is parallel with the anterior aspect of the tibia and the knee is flexed at $20^{\circ}$. The cassette is holed by the patient at $90^{\circ}$ against the beam.

3. Merchant: The patient stays in supination on the radiological table with the knees flexed at $90^{\circ}$ and the cassette is placed proximal from the shins. Both knees are exposed simultaneously with the beam directed to the legs, tilted at $30^{\circ}$ from the horizontal.

\section{The patellofemoral incidence in axial plane with or without weight bearing}

The views described previously are done without weight bearing. The outcome of the extensor mechanism to the patellofemoral alignment is not taken into account in this radiological view. An axial weight bearing position that respects the entire Merchant incidences parameters is described and approved [39]. This is achieved with the patient standing against a wall (in front of the mobile radiological support) at $25.4 \mathrm{~m}$ (10 inch) distance to the wall. The beam source is brought to the level of the head of the patient. The $18 \times 43 \mathrm{~cm}$ cassette is positioned on the dorsal aspect of the legs, which will stay parallel one against the other. The knee is flexed $45^{\circ}$, with the tibia at $15^{\circ}$ from the beam. This radiological view shows the patellofemoral maltracking, compared to the standard Merchant's view: the lateral tilt and the subluxation of the substituted patella are reduced significantly, the prevalence of the uncovered patella towards femoral trochlear impingement is significantly increased, and the patellar maltracking is much easier to correlate with the clinical symptoms. Using the weight bearing axial view for the evaluation of the total knee arthroplasty offers additional information over the standard radiography.

\section{Patellofemoral axis evaluation}

The patellofemoral alignment, measured according to the $\mathrm{KS}$ guide takes into account the following: the thickness of the patella, the width, the tilt, the medio-lateral tracking, and the patellar prosthesis angle to the patellar bone. Those measurements are calculated according to Gomes and Gustilo [40].

1. The preoperative patellar tilt or the unsurfaced patella is formed by a line dragged anterior to the condylar or prosthetic limit in the axial line and a line that connects the ape of the patellar articular surface with the lateral edge of the lateral facet of the patella. If the angle is positive, this is considered normal. If the angle is o or negative, the patellar tilt is abnormal. A small or negative angle indicates a subluxation or a dislocation.

The patellar tilt after patellar prosthesis is an angle formed by a line that passes anterior to the femoral condyles and a line through the bone-prosthesis interface.

2. The pre and postoperative thickness of the patella is the vertical distance from the anterior cortex of the patella to the patellar femoral sulcus. A study on cadavers by Scott shows the flexion decreases by $3^{0}$ for every 2 $\mathrm{mm}$ incrementation of the patellar thickness [41].

3. The medial-lateral position of the patella is the horizontal distance between two vertical lines perpendicular on a line that goes through the femoral condyles. A line is formed by the middle of the patella and another line by the patellar femoral sulcus.

\section{The axial femoral incidence}

Besides the patellofemoral evaluation, an axial view offers information referring to the position of the femoral component in axial plane. The femoral component rotation is calculated after the condylar twisted angle (CTA), formed between posterior condylar axis and the clinical epicondylar axis. This angle can be obtained using a CAT scan but using simple radiography as well $[42,43]$. Preoperative use 
of such incidence may anticipate the grades of external rotation from the posterior condyles, which the surgeon can use to obtain a correct positioning, identical with the one obtained using the trans-epicondylar axis (TEA).

1. The Takai view - the patient positioning must be in such way that the affected knee is bent at $80^{\circ}$ and holed by a support (chair). The $\mathrm{X}$-ray beam is perpendicular on the tibial shaft. The radiology resulted after this positioning is a posterior-lateral view of a flexed knee.

2. Kanekasu view - the patient is positioned on a framework with the lower limbs suspended and the knees flexed at $90^{\circ}$. The authors of this view have modified the original position, adding a $1.5 \mathrm{~kg}$ distraction on the affected leg, being able to evaluate the tibiofemoral space configuration preoperative and postoperative [44].

\section{CT protocol}

The axial plane evaluation for total knee arthroplasty may be done by using CT scan or MRI. The CT examination is used most frequently. Berger created a protocol for the evaluation of the transversal (axial) plane rotation $[45,46]$.

\section{The Berger protocol}

The patient stays in supination, with the examined limb in full extension, so the scanning will go perpendicular on the anatomical axis of the knee. In the lateral, scout view, the $\mathrm{CT}$ incidences are done perpendicular on the femoral axis for the femoral scanning and perpendicular on the tibial axis for tibial scanning. The $1.5 \mathrm{~mm}$ thickness $C T$ sections are necessary in four locations: through epicondylar axis of the femur, the tibial tubercle, superior edge of the plateau and the tibial component.

\section{The femoral component rotation}

The optimal level to view both femoral epicondyles is at $30 \mathrm{~mm}$ proximal from the joint line [47]. Two lines are drawn at this level: a line tangent to the posterior condyles, the second line connects the lateral epicondyle prominence with the medial epicondyle sulcus (surgical TEA = transepicondylar axis). The angle formed by those two lines is the posterior condylar angle (PCA). The value of this angle must be as close to $0^{0}$ as possible. Romero et al. suggests using TEA, which connects the lateral epicondyles with the edge of the medial epicondyle, being a much easier landmark to find [47]. This axis is helpful to calculate the CTA (condylar twist angle) (the angle between the posterior condylar axis and the clinical transepicondylar axis) that has $3-4^{\circ}$ of external rotation by the PCA [47].

\section{The tibial component rotation}

The geometrical center of the proximal tibial and its axial transposition above the tibial tubercle must be determined for the evaluation of the tibial component rotation [48]. After that, the geometrical center of the proximal tibial component and the junction between medial edge and the medial third of the tibial tubercle are connected, resulting the tubercle orientation. The anteroposterior axis of the tibial component is evaluated on the axial view through the tibial component. The rotation of the tibial component is calculated in relationship with the tibial tubercle orientation and the anteroposterior axis of the tibial component [48].

\section{The Akagi line}

The line, which goes from the insertion center of the posterior cruciate ligament towards the medial aspect of the patellar ligament, must be perpendicular on the surgical transepicondylar axis of the femur, on the transversal plane CT image [49].

\section{The Insall line}

The central anteroposterior axis of the tibial component has to coincide with the line, which connects the $P C L$ insertion to the junction between $1 / 3$ medial to $2 / 3$ lateral of the patellar ligament. 


\section{Conclusion}

Sagittal and axial roentgenographic analysis for the preoperative planning and after TKA are important steps, however, they have a limit: they are bidimensional. The understanding of rotational assessment of components, combined components position under load, kinematics relationships between components and between bones, will be possible only when the $3 \mathrm{D}$ evaluation of the entire lower limb will be made on a routine basis.

\section{References}

1. Pavlov H. Lower Extremity, Knee. Lateral view. In: Pavlov H, Burke M, Giesa M et al. Orthopaedist's guide to plain film imaging. 1999, New York, Thieme.

2. Berquist TH. The Knee. In: Berquist TH. Imaging of Orthopaedic Fixation Devices and Prostheses. 2009, Philadelphia, Lippincott Williams \& Wilkins, 251-332.

3. Paley D. Radiographic Assessment of Lower Limb Deformities. In: Paley D. Principles of Deformity Correction. 2002, New York, Springer.

4. Minoda Y, Kobayashi A, Iwaki H et al. TKA sagittal alignment with navigation system and conventional technique vary only few degrees. Clin Orthop Relat Res. 2009; 467:1000-1006.

5. Fang DM, Ritter MA, Davis KE et al. Coronal alignment in total knee arthroplasty: Just how important is it? J Arthroplasty. 2009; 24(6, suppl):39-43.

6. Yehyawi TM, Callaghan JJ, Pedersen DR et al. Variances in Sagittal Femoral Shaft Bowing in Patients Undergoing TKA. Clin Orthop Relat Res. 2007; 464:99-104.

7. Chung BJ, Kang YG, Chang CB et al. Differences between sagittal femoral mechanical and distal reference axes should be considered in navigated Res. 2009; 467:240 3-13.

8. Seo JG, Kim BK, Moon YW et al. Bony landmarks for determining the mechanical axis of the femur in the sagittal plane during total knee arthroplasty. Clin Orthop Surg. 2009; 1:128-31.

9. Balakrishnan V, De Steigert R, Lowe A. Radiographic assessment of alignment following TKA: outline of standardized protocol and assessment of a newly devise trigonometric method of analysis. ANZ J Surg. 2010; 80: 344-9.

10. Hankemeier S, Gosling T, Richter $\mathrm{M}$ et al. Computer assisted analysis of lower limb geometry: higher intra observer reliability compared to conventional method. Comput Aided Surg. 2006; 11:81-6.

11. Hinman RS, May RL, Crossley KM. Is there an alternative to the full leg radiograph for determining knee joint alignment in osteoarthritis?. Arthritis Rheum. 2006; 55:306-13.

12. Yin L, Chen K, Guo L et al. Knee alignment in the transverse plane during weight-bearing activity and its implication for the tibial rotational alignment in total knee arthroplasty. Clin Biomech (Bristol, Avon). 2015; 30(6):565-71.

13. Maderbacher G, Baier C, Benditz A et al. Presence of rotational errors in long leg radiographs after total knee arthroplasty and impact on measured lower limb and component alignment. Int Orthop. 2017 Jan 31.

14. Yoo JH, Chang CB, Shin KS et al. Anatomical references to assess the posterior tibial slope in total knee arthroplasty: a comparison of 5 anatomical axes. J Arthroplasty. 2008; 23:586-92.

15. Denis K, Van Ham G, Bellemans J et al. How correctly does an intramedullary rod represent the longitudinal tibial axes?. Clin Orthop Related Res. 2002; 397:424-33.

16. Singh G, Tan JH, Sng BY et al. Restoring the anatomical tibial slope and limb axis may maximize post-operative flexion in posterior-stabilized total knee replacements. Bone Joint J. 2013; 95-B(10): 1354-1358.

17. Bellemans J, Banks S, Victor J, et al. Fluoroscopic analysis of the kinematics of deepflexion in total knee arthroplasty. J Bone Joint Surg Br. 2002; 84: 50-3.

18. Soda Y, Oishi J, Nakasa T et al. New parameter of flexion after posterior stabilized total knee arthroplasty: posterior condylar offset ratio on X-ray photographs. Arch Orthop Trauma Surg. 2007; 127:167-70.

19. Arabori M, Matsui N, Kuroda R et al. Posterior condylar offset and flexion in posterior cruciate-retaining an posterior stabilized TKA. J Orthop Sci. 2008; 13:46-50.

20. Clarke HD, Hentz JG. Restoration of femoral anatomy in TKA with unisex and gender-specific components. Clin Orthop Relat Res. 2008; 466:2711-6.

21. Fehring TK, Odum SM, Hughes J et al. Differences between the sexes in the anatomy of the anterior condyle of the knee. J Bone Joint Surg Am. 2009; 91:2335-41.

22. Poilvache PL, Insall JN, Scuderi GR et al. Rotational landmarks and sizing of the distal femur in total knee arthroplasty. Clin Orthop Relat Res. 1996; (331):35-46.

23. WeissmanBN. Radiographic evaluation of total joint replacement. In: Sledge CB, Ruddy S, Harris ED Jr. et al. Arthritis Surgery. 1994, Philadelphia, PA, WB Saunders, 846-907.

24. Miller RK, Goodfellow JW, Murray DW et al. In vitro measurement of patellofemoral force after three types of knee replacement. J Bone Joint Surg Br. 1998; 80:900.

25. Caton JH, Prudhon JL, Aslanian T et al. Patellar height assessment in total knee arthroplasty: a new method. Int Orthop. 2016; 40(12):2527-2531.

26. Lin CF, Wu JJ, Chen TS et al. Comparison of the Insall-Salvati ratio of the patella in patients with and without an ACL tear. Knee Surg Sports Traumatol Arthrosc. 2005; 13(1):8-11.

27. Allen AM, Ward WG, Pope TL et al. Imaging of the total knee arthroplasty. Radiol Clin North Am. 1995; 33:289303. 
28. Eshnazarov KE, Seon JK, Song EK. Comparison of Radiological Assessments Patellar Resurfacing with Retention for Grade IV Osteoarthritis in Patellofemoral Joint accomplished Total Knee Arthroplasty. Vestn Rentgenol Radiol. 2016; 97(1):28-32.

29. Malo M, Vince KG. The unstable patella after total knee arthroplasty: Etiology, prevention, and management. J Am Acad Orthop Surg. 2003; 11(5):364-371.

30. Aglietti P, Baldini A, Buzzi R et al. Patella resurfacing in total knee replacement: functional evaluation and complications. Knee Surg Sports Traumatol Arthrosc. 2001; 9:S27-33.

31. Figgie H, Goldberg V, Heiple K et al. The influence of tibial-patellofemoral location on function of the knee in patients with posterior stabilized condylar knee prosthesis. J Bone Joint Surg. 1986; 68:1035.

32. Kawamura H, Bourne RB. Factors affecting range of flexion after total knee arthroplasty. J Orthop Sci. 2001; 6:248-52.

33. Selvarajah E, Hooper G. Restoration of the joint line in total knee arthroplasty. J Arthroplasty. 2009;24:10991102.

34. Mihalko WM, Boyle J, Clark LD et al. The variability of intramedullary alignment of the femoral component during total knee arthroplasty. J Arthroplasty. 2005; 20:25-8.

35. Price AJ, Rees JL, Beard DJ et al. Sagittal plane kinematics of a mobile-bearing unicompartimental knee arthroplasty at 10 years: a comparative in vivo fluoroscopic analysis. J Arthroplasty. 2004; 19: 0-7.

36. Chia SL, Merican AM, Devadasan B et al. Radiographic features predictive of patellar maltracking during total knee arthroplasty. Knee Surg Sports Traumatol Arthrosc. 2009; 17(10):1217-1224.

37. Classen T, Wegner A, Muller RD et al. Femoral component rotation and Laurin angle after total knee arthroplasty. Acta Orthop Belg. 2010; 76(1):69-73.

38. Huang TW, Hsu WH, Peng KT et al. Total knee replacement in patients with significant femoral bowing in the coronal plane: a comparison of conventional and computer-assisted surgery in an Asian population. J Bone Joint Surg Br. 2011; 93:345-50.

39. Baldini A, Anderson JA, Cerulli-Mariani $\mathrm{P}$ et al. Patellofemoral evaluation after total knee arthroplasty. Validation of a new weight-bearing axial radiographic view. J Bon Joint Surg Am. 2007; 89 1810-7.

40. Merican AM, Ghosh KM, Baena FR et al. Patellar thickness and lateral retinacular release affects patellofemoral kinematics in total knee arthroplasty. Knee Surg Sports Traumatol Arthrosc. 2014; 22(3):526-533.

41. Bengs BC, Scott RD. The effect of patellar thickness on intraoperative knee flexion and patellar tracking in total knee arthroplasty. J Arthroplasty. 2006; 21:650-5.

42. Kaneka su K, Kondo M, Kadoya Y. Axial radiography of the distal femur to assess rotational alignment in total knee arthroplasty. Clin Orthop Relat Res. 2005; (434):193-7.
43. Takai S, Yoshino N, Isshiki T et al. Kneeling view: a new roentgenographic technique to assess rotational deformity and alignment of the distal femur. J Arthroplasty. 2003; 18:478-83.

44. Tokuhara Y, Kadoya Y, Kanekasu K et al. Evaluation of the flexion gap by axial radiography of the distal femur. J Bone Joint Surg Br. 2006; 88:1327-30.

45. Crossett LS, Rubash HE, Berger R. Computerized tomography in total knee arthroplasty. In: Insall JN, Scott WN, Scuderi GR. Current Concepts in Primary and Revision Total Knee Arthroplasty. 1996, Philadelphia, PA, Lippincott- Raven, 235-248.

46. Berger RA, Seel MJ, Schleiden $M$ et al Determination of femoral component rotation in total knee arthroplasty using computer tomography. Orthop Trans. 1993; 17:427.

47. Suter T, Zanetti M, Schmid M et al. Reproducibility of measurement of femoral component rotation after total knee arthroplasty using computer tomography. J Arthroplasty. 2006; 21:744-8.

48. De Valk EJ, Noorduyn JC, Mutsaerts EL. How to assess femoral and tibial component rotation after total knee arthroplasty with computed tomography: a systematic review. Review article. Knee Surg Sports Traumatol Arthrosc. 2016; 24(11):3517-3528.

49. Yu HC, Wen H, Zhang Y et al. Research on the reliability of the Akagi line as a reference axis to guide for rotational alignment of the proximal tibial component in total knee arthroplasty. Zhongguo Gu Shang. 2015; 28(10):884-7. 